\title{
Reliability analysis of a retrial queueing systems with collisions, impatient customers, and catastrophic breakdowns
}

\author{
Attila Kuki, Tamás Bérczes, János Sztrik, Ádám Tóth \\ Faculty of Informatics University of Debrecen, Hungary,4028 \\ \{kuki.attila, berczes.tamas, janos.sztrik, toth.adam\}@inf.unideb.hu
}

\begin{abstract}
A lot of different real-life systems can be modeled by retrial queuing $(R Q)$ models. In this paper, RQ-systems are considered. The single server system is non-reliable, nondeterministic system failures might occur. This is a finite source system. In applications, it is more realistic, and there is no stability problem. One of the first considered system operational characteristics is the collision or the conflict of customers. When a job is under service at the server, and a new job comes, they will collide. In this case, both jobs will transport to a virtual waiting room, called orbit. The customers retry their requests from the orbit. The retrial times are random. Server failures might happen, the server might go down. While the server is down state, the new requests are transported to the orbit, or the source is blocked, that is, no customer can enter into the system. The second system characteristic is the impatient property of the customers. The customers stay in the orbit and waiting for their service. After a non-deterministic time-interval, a customer gives up retrying and leaves the system. These customers will be lost from the system, they remain unserved. This is the impatient characteristic. The third system characteristic is the catastrophic breakdown. It means, that in case of a negative event, all of the customers at the server and in the orbit leave the system, and take their places in the source. The novelty of this paper is to investigate the phenomenon of the catastrophic breakdown in a collision environment with impatient customers.

This impatient property results, that the recursive algorithm for the time-independent probabilities can not be formulated. MOSEL-2 tool can be used for solving the system equations and calculating the system performance measures. These measures are, for example, the average sojourn time and other reliability metrics. The main goal is to investigate the effect of the impatient property under catastrophic breakdown. Numerical results are presented graphically, as well.
\end{abstract}

\section{INTRODUCTION}

Modeling infocommunication systems is essential to understand their dynamical behavior and find an optimal working environment. There exist a lot of tools and methods for modeling these types of systems. One of the most popular and effective tools are the retrial queueing systems (RQ-systems). Compared with the simple queueing systems, in RQ-models the customers are not lost when the system is busy. An RQsystem can have an infinite and finite number of sources. The requests arrive from the outside world or the source facility, respectively. If a customer arrives to the server, and the server is still working with an other customer, the new job enters a virtual lobby, called orbit and waits for a non-deterministic time interval (exponential), and it retries its service demand again. The orbit can be imagined as a virtual waiting facility, and it is assumed to be large enough, so an incoming job always finds a free place in the orbit. From the orbit, the jobs do not give up, they try to reach the server. Once the server is free, the jobs step into the service facility, and they will be served. This is the patient behavior of jobs.

In this paper, the impatient behavior of the customers is also considered. A job waiting in the virtual lobby (orbit) might leave the system after constant or random waiting time. Our models deal with random times, described with a distribution, e.g. the exponential distribution. These customers will remain unserved, their requests are lost.

Examples of technologies and applications, which can be modeled by an RQ-systems can be telephone centers, sensor networks, repair facilities, telecommunication environment, etc. Infinite source models have been considered and studied by numerous authors, very large number of papers were published in the literature. But, in real-life applications, there are a lot of situations, where the infinite sources are not so suitable. When there are only finite numbers of entities, which are related with the system, the finite source models are more adequate. Many examples can be mentioned, for example, mobile cellular networks, intelligent sensor networks, a lot of new IoT systems, and so on. Results on finite source retrial queueing systems are, for example, in [1], [2], [3], [4], [5], [6].

In many applications, unfortunately, the systems are nonreliable. They subject to random breakdowns. This situation also has to be investigated. Random server failures and repairs are included in the models. Generally or exponentially distributed random times and time intervals can be considered in modeling the breakdown and repair processes. A non-reliable system is very sensitive, the calculated outcomes and descriptors of the system have to be handled very carefully. Nonreliable, finite source retrial systems have been investigated by many authors, e.g. in [7], [8], [9], [3], [10].

A non-reliable $M / M / 1 / / N$ retrial queueing system with conflict of jobs is also a part of this investigation. The phenomenon of collisions of jobs (or conflict of jobs) is a very common behavior in non-synchronized infocommunication systems with a constrained resources, for example, Ethernet transmissions and other communication facilities. In case of 
conflict, all of the involved signals are damaged, and they need to re-send. The working and the performance of the system is sub-optimal. It is very important, to build up procedures, which try to avoid or at least decrease the effects of conflicts. Results can be found in [11], [12], [13], [14], [15], [16].

The focus of this paper is the catastrophic breakdown. Retrial queueing models in which customers are removed from the system due to catastrophic or disaster events have been investigated extensively by authors. Modeling special systems, e.g. automatic teller machines needs different types of breakdowns. A catastrophic event can be, for example, mechanical failures or power outages. Disaster events are known also as a negative arrival or a negative customer. The presence or arrival of a negative job might be dangerous for the system. The other jobs, which work for the system, are called positive jobs. These positive jobs can be damaged by the negative ones. The positive jobs might even be removed from the system. The most extreme situation is, when all of the positive jobs are removed from the system. This event is called a disaster, or catastrophic breakdown. In addition, this type of event interrupts all of the service processes at the servers and breaks down the service unit. The service in the service unit will be interrupted. The service has been done until the interruption point is lost. All of the customers from the server and the orbit are sent back to the source. Detailed studies on catastrophic breakdowns and negative customers can be found in [17], [18] [19], [20], [21].

The impatient property of customers and the feature of catastrophic breakdown make the system equations so complex, that a simple numeric solution can not be performed. That's why a computer program is used for calculating the system probabilities. Using the resulting steady-state system distribution, the most important system measures characteristics can be formulated. Figures will be provided with the effect of different parameters on these performance metrics.

\section{DESCRIPTION OF THE SYSTEM}

In this paper, an $M / M / 1 / / N$ model is considered. This is a finite source system, and by Kendall's description, the interarrival times and the service times follow the exponential law, the number of servers is one, and the number of sources is $N$. Two scenarios of the system can be studied and compared:

- The common break-down mode. This is the well-known non-reliable environment, that is the service facility is exposed to non-deterministic failures. The inter-event times between the failures follow the exponential law. Two different parameters can be considered for describing the failure events. In the case of a non-working service unit, the parameter is $\gamma_{0}$. In the case of a working server, the failure parameter is $\gamma_{1}$. Later, in numerical investigations, these two parameters will not be distinguished. The behavior of the job interrupted at the server can be different. The service can be continued or started again. Here, the job interrupted at the service facility is transferred back to the orbit. There is no time transition before the repair. After the failure event, the process of the repair begins immediately. The repair time is also a random variable, the distribution is exponential, and the repair parameter is $\gamma_{2}$. While the repair is in progress, different behavior of the source can be handled. The system is blocked, that is, no new request can enter into the system. Or, the system is not blocked, generating of new requests continues. These new jobs can not reach the server under repair, so they are transferred into the orbit. Since this is a retrial system, the jobs try to find a free server from the orbit. This retrial is described with a random variable with exponential distribution. The retrial parameter is $\sigma / N$. The customers do not give up finding a free server in up state. In the models considered below, the blocked situation will be studied.

- The catastrophic break-down mode. This is the situation when a disaster event removes all of the jobs from the system (from the orbit and from the server after interrupting the service). The repair of the system starts immediately. The same breakdown parameters are used as in the common breakdown mode, i.e. $\gamma_{0}$ and $\gamma_{1}$ for an idle server breakdown and a busy server breakdown, respectively, and $\gamma_{2}$ for the repair. During the down period of the system, the source is blocked, no new request can enter into the system.

- The customers have impatient behavior. The breakdown event of the server is either the regular (common) breakdown mode, or it is the catastrophic breakdown. The customers are impatient. Based on the retrial property, a customer retries to reach the server after a random, exponentially distributed time, or the customer gives it up, and leaves the service unit, and is transferred back to the source after a random waiting time. The distribution is exponential, and the parameter is $\tau$. In this case, the customer will not be served.

Let us consider the dynamic workflow of the system. A job (it can be called a customer or a request, as well) is generated from the source. The job inter-arrival times toward the server are exponentially distributed random times with parameter $\lambda / N$. Until the end of service of this job, the source will not generate a new job for this token. The generated job enters the service environment. The server can be empty (or idle), or there can be another job under service. This time the server is called as busy. When there is no job at the server, the service of the incoming job, which can arrive from the source or the orbit, starts at once. The random service time intervals are considered to follow the exponential distribution law with parameter $\mu$. When the server is in busy state, that is a job is under service at the server, and a new request is arriving to the server, the two jobs will collide. This is the phenomenon of the collision of the customers. In this case, both jobs (the job served and the job just arrived) are moved into the orbit. From the orbit the jobs retry reaching the server again after a random time interval. The distribution of the retrial times is exponential with the expectation of $1 /(\sigma / N)$. See the model on Figure 1.

Let's consider the following notations. $i(t)$ denotes the state of the system. This is the total number of jobs in the system (under service and waiting in the orbit). $k(t)$ is the server 
status descriptor:

$k(t)=\left\{\begin{array}{l}0, \text { the service unit is up and non-working } \\ 1, \text { the service unit is up and working } \\ 2, \text { the service unit is failed and repair is in progress. }\end{array}\right.$

The $P(k(t)=k, i(t)=i)=P_{k}(i, t)$ quantities are the system probabilities: at a time of $t$ there are number of $i$ jobs in the system and the value of the server state descriptor is $k$. With these conditions the process $X(t)=\{k(t), i(t)\}$ is a Markovian-chain with two dimensions, and a state space of $\{0,1,2\} x\{0,1, \ldots, N\}$.

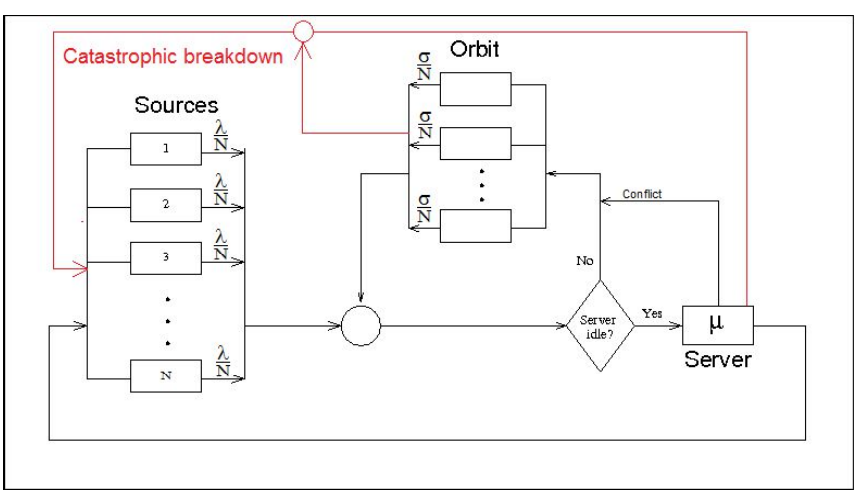

Fig. 1. System model

A successfully served job steps back to the source. All the random times, time intervals considered in the model are assumed to be totally independent of each other.

Since the $X(t)=\{k(t), i(t)\}$ process is a finite state Markov-chain, so the stability conditions hold, and the existence of the steady-state probabilities of $P_{k}(i, t)=P_{k}(i)$ is ensured.

For patient case, and with collision of jobs, the Kolmogorov balance equations for probabilities $P_{k}(i, t)$ can be written, as follows (see, in [13] and [14]):

$$
\begin{gathered}
\frac{\partial P_{0}(0, t)}{\partial t}=-\left(\lambda+\gamma_{0}\right) P_{0}(0, t)+\mu P_{1}(1, t)+\gamma_{2} P_{2}(0, t) \\
\frac{\partial P_{1}(1, t)}{\partial t}=-\left(\lambda \frac{N-1}{N}+\mu+\gamma_{1}\right) P_{1}(1, t)+ \\
+\lambda P_{0}(0, t)+\frac{\sigma}{N} P_{0}(1, t) \\
\quad \frac{\partial P_{2}(0, t)}{\partial t}=-\left(\lambda+\gamma_{2}\right) P_{2}(0, t)+\gamma_{0} P_{0}(0, t) \\
\frac{\partial P_{0}(i, t)}{\partial t}=-\left(\lambda \frac{N-1}{N}+\sigma \frac{i}{N}+\gamma_{0}\right) P_{0}(i, t)+ \\
+\mu P_{1}(i+1, t)+\lambda \frac{N-i+1}{N} P_{1}(i-1, t)+ \\
+\sigma \frac{i-1}{N} P_{1}(i, t)+\gamma_{2} P_{2}(i, t)
\end{gathered}
$$

$$
\begin{aligned}
& \frac{\partial P_{1}(i, t)}{\partial t}=-\left(\lambda \frac{N-1}{N}+\sigma \frac{i-1}{N}+\gamma_{1}+\mu\right) P_{1}(i, t)+ \\
& +\lambda \frac{N-i+1}{N} P_{0}(i-1, t)+\sigma \frac{i}{N} P_{0}(i, t), \\
& \frac{\partial P_{2}(i, t)}{\partial t}=-\left(\lambda \frac{N-1}{N}+\gamma_{2}\right) P_{2}(i, t)+\gamma_{0} P_{0}(i, t)+ \\
& +\gamma_{1} P_{1}(i, t)+\lambda \frac{N-i+1}{N} P_{2}(i-1, t) .
\end{aligned}
$$

Again, the $X(t)=\{k(t), i(t)\}$ process is a finite state Markov-chain, so the stability conditions hold, and the existence of the steady-state probabilities of $P_{k}(i, t)=P_{k}(i)$ is ensured.

Thus, the steady-state Kolmogorov balance equations can be formulated, as

$$
\begin{gathered}
-\left(\lambda+\gamma_{0}\right) P_{0}(0)+\mu P_{1}(1)+\gamma_{2} P_{2}(0)=0 \\
-\left(\lambda \frac{N-1}{N}+\mu+\gamma_{1}\right) P_{1}(1)+\lambda P_{0}(0)+\frac{\sigma}{N} P_{0}(1)=0 \\
-\left(\lambda+\gamma_{2}\right) P_{2}(0)+\gamma_{0} P_{0}(0)=0 \\
-\left(\lambda \frac{N-1}{N}+\sigma \frac{i}{N}+\gamma_{0}\right) P_{0}(i)+\mu P_{1}(i+1)+ \\
+\lambda \frac{N-i+1}{N} P_{1}(i-1)+\sigma \frac{i-1}{N} P_{1}(i)+\gamma_{2} P_{2}(i)=0 \\
-\left(\lambda \frac{N-1}{N}+\sigma \frac{i-1}{N}+\gamma_{1}+\mu\right) P_{1}(i)+ \\
+\lambda \frac{N-i+1}{N} P_{0}(i-1)+\sigma \frac{i}{N} P_{0}(i)=0 \\
\quad-\left(\lambda \frac{N-1}{N}+\gamma_{2}\right) P_{2}(i)+\gamma_{0} P_{0}(i)+ \\
+\gamma \frac{P}{1}(i)+\lambda \frac{N-i+1}{N} P_{2}(i-1)=0
\end{gathered}
$$

From these equations, the case of a reliable server with collision of customers can be derived easily. Just give the value of zero for the parameter of $\gamma_{2}$, and probabilities of $P_{2}$.

Following a similar method, the system balance equations can be formulated for the case of collision, server with regular failure, and impatient jobs:

$$
\begin{gathered}
-\left(\lambda+\gamma_{0}\right) P_{0}(0)+\mu P_{1}(1)+\gamma_{2} P_{2}(0)+\frac{\tau}{n} P_{0}(1)=0 \\
-\left(\lambda \frac{N-1}{N}+\mu+\gamma_{1}\right) P_{1}(1)+\lambda P_{0}(0)+\frac{\sigma}{N} P_{0}(1)+ \\
+\frac{\tau}{n} P_{1}(2)=0 \\
-\left(\lambda+\gamma_{2}\right) P_{2}(0)+\gamma_{0} P_{0}(0)=0
\end{gathered}
$$




$$
\begin{aligned}
& -\left(\lambda \frac{N-i}{N}+\sigma \frac{i}{N}+\tau \frac{i}{N}+\gamma_{0}\right) P_{0}(i)+\mu P_{1}(i+1)+ \\
& +\lambda \frac{N-i+1}{N} P_{1}(i-1)+\sigma \frac{i-1}{N} P_{1}(i)+ \\
& +\tau \frac{i+1}{N} P_{0}(i+1)+\gamma_{2} P_{2}(i)=0 \\
& -\left(\lambda \frac{N-i}{N}+\sigma \frac{i-1}{N}+\tau \frac{i-1}{N}+\gamma_{1}+\mu\right) P_{1}(i)+ \\
& +\lambda \frac{N-i+1}{N} P_{0}(i-1)+\sigma \frac{i}{N} P_{0}(i)+\tau \frac{i}{N} P_{1}(i+1)=0 \\
& -\left(\lambda \frac{N-i}{N}+\gamma_{2}\right) P_{2}(i)+\gamma_{0} P_{0}(i)+\gamma_{1} P_{1}+ \\
& +\lambda \frac{N-i+1}{N} P_{2}(i-1)+\tau \frac{i+1}{N} P_{2}(i+1)=0 .
\end{aligned}
$$

\section{Performance Characteristics}

Investigating the effect of the different parameters on the behavior of the system, the usual performance characteristics can be calculated from the steady-state probabilities.

- Average number of customers in the system $\bar{Q}$ and in the orbit $\bar{O}$

$$
\bar{Q}=\sum_{i=0}^{N} i P(i), \quad \bar{O}=\bar{Q}-P_{1}
$$

- Average arrival rate $\bar{\lambda}$

$$
\bar{\lambda}=\sum_{k=0}^{1} \sum_{i=0}^{N}(N-i) \frac{\lambda}{N} P_{k}(i)
$$

- Average response time $\bar{T}$ and mean waiting time $\bar{W}$ in the orbit can be obtained by the Little-formula

$$
\begin{gathered}
\bar{T}=\frac{\bar{Q}}{\bar{\lambda}}, \quad \bar{W}=\frac{\bar{O}}{\bar{\lambda}}, \\
\bar{O}=\bar{Q}-P_{1},
\end{gathered}
$$

- Average total service time $E\left(T_{S}\right)$ and average total sojourn time in the source $E(\kappa)$

$$
E\left(T_{S}\right)=\bar{T}-\bar{W}, \quad E(\kappa)=\frac{(N-\bar{Q}) \bar{T}}{\bar{Q}},
$$

- Average number of trials from the source $E\left(N_{T S}\right)$ and from the orbit $E\left(N_{T O}\right)$

$$
\begin{array}{cc}
E\left(N_{T S}\right)=\frac{\lambda}{N} E(\tau), & E\left(N_{T O}\right)=\frac{\sigma}{N} \bar{W} . \\
\bar{Q}=\sum_{i=0}^{N} i P(i), \quad \bar{O}=\bar{Q}-P_{1} .
\end{array}
$$

\section{NUMERICAL RESULTS}

The steady-state equations can be solved by different methods. Here an analytical software tool, namely the MOSEL-2 was chosen. This tool formulates the underlying Markovianequations of the system, and provides an algebraic solution for the steady-state probabilities. With the assumption of exponentiality of the system parameters, this tool is effective and quick for a reasonably large number of sources.

\begin{tabular}{|c|c|c|c|c|c|c|}
\hline Figure & $\boldsymbol{\lambda}$ & $\boldsymbol{\mu}$ & $\boldsymbol{\sigma}$ & $\boldsymbol{\tau}$ & $\boldsymbol{\gamma}_{\mathbf{0}}=\boldsymbol{\gamma}_{\mathbf{1}}$ & $\boldsymbol{\gamma}_{\mathbf{2}}$ \\
\hline $\mathbf{2}$ & $0.1 . .10$ & 1 & 5 & Legend & 0.01 & 1 \\
\hline $\mathbf{3}$ & $03.7 . .7 .7$ & 1 & 5 & Legend & 0.01 & 1 \\
\hline $\mathbf{4}$ & $0.1 . .10$ & 1 & 5 & Legend & 0.01 & 1 \\
\hline $\mathbf{5}$ & 1 & 1 & 5 & Legend & $0.001 . .0 .111$ & 1 \\
\hline $\mathbf{6}$ & 1 & 1 & 5 & Legend & $0.001 . .0 .111$ & 1 \\
\hline $\mathbf{7}$ & 1 & 1 & 5 & Legend & $0.001 . .0 .111$ & 1 \\
\hline
\end{tabular}

TABLE I

NUMERICAL VALUES OF MODEL PARAMETERS

The collision of customers and catastrophic breakdown features are applied for all of the following figures. In the MOSEL program the idle and the busy state failure rates $\left(\gamma_{0}\right.$ and $\gamma_{1}$ are the same. The number of sources is $N=100$. All the other system parameters are listed in Table I.

The dynamic behavior of the system can be seen on the figures. Different system characteristics are displayed in function of overall generation rate and the failure rate.

All figures compare three different cases with respect to different values of the impatient parameter, $\tau$. The first one is a small parameter value. In this case, the expectation of patient time interval is large. This case corresponds to the patient behavior of the customers. The other two values of the impatient parameter are medium and large.

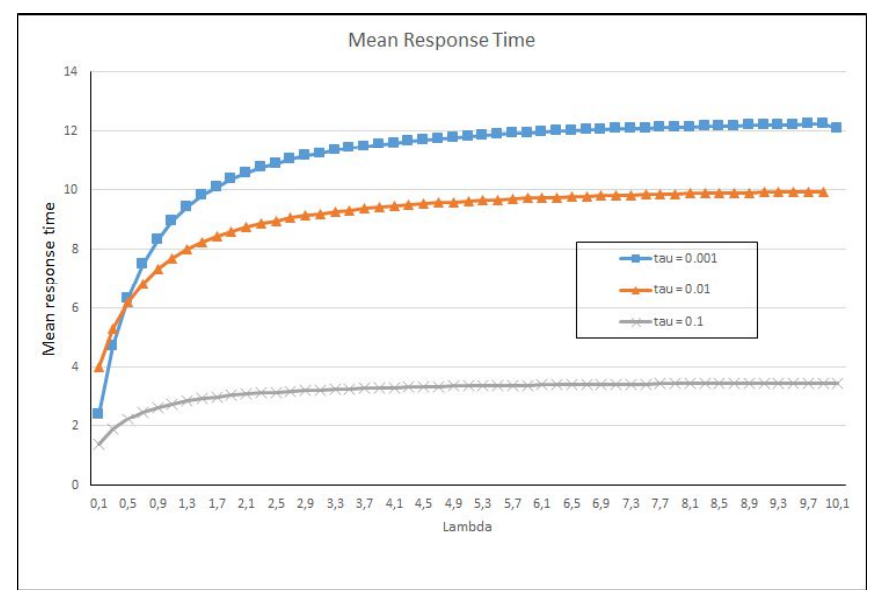

Fig. 2. Mean response time vs. generation rate

On Figures 2, 3, and 4 the overall generation rate, $\lambda$ is the running parameter on the $\mathrm{X}$-axes. On Figure 2 the mean response time is displayed. For the patient behavior, this performance measure has larger values. In this case, the customers do not leave the system from the orbit.

Figure 3 shows the utilization of the server. For larger generation rates larger utilization can be observed. It is interesting, that for larger impatient parameters larger increments of the 


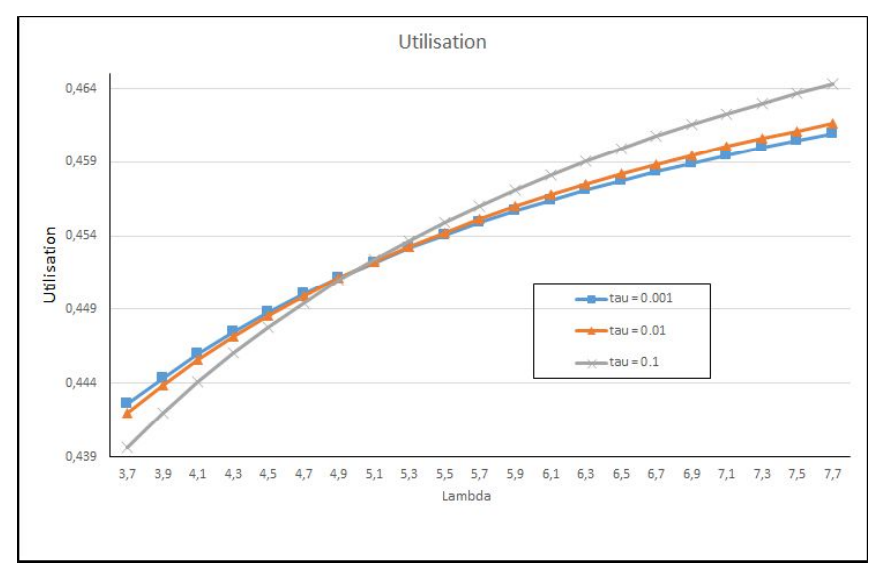

Fig. 3. Utilization vs. generation rate

corresponding curves are present. In addition, the curves have a single intersection point, which means, for a given generation rate $(\lambda=5)$, the same utilization can be observed with a different impatient rate. This phenomenon might be resulted from a special coincidence of the collision, the catastrophic breakdown, and the impatient properties.

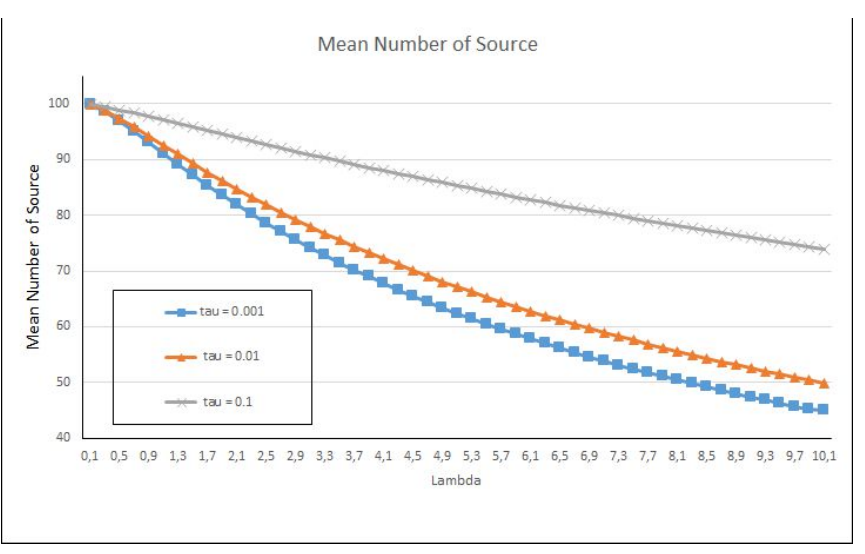

Fig. 4. Mean number of source vs. generation rate

On Figure 4 the number of free tokens in the source can be seen. The curves have a decrement in their slopes. The higher generation rate implies a higher number of collisions, so, the customers will fill up the orbit. The patient customers remain in the orbit, consequently, the corresponding curve has the smallest values.

On Figures 5, 6, and 7 the failure rates are the running parameter. The figures display the same system characteristics, as Figures 2, 3, and 4. Due to the increasing catastrophic failure generation rates, the mean response time and the utilization have decreasing curves. Because of the frequent catastrophic breakdown, all of the customers leave the systems at every breakdown event, thus the number of sources will increase. These general trends are refined with the patient / impatient behavior of the customer.

\section{CONCLUSION}

In this paper, the interaction of the behaviors of collision, catastrophic breakdown, and impatient customers has

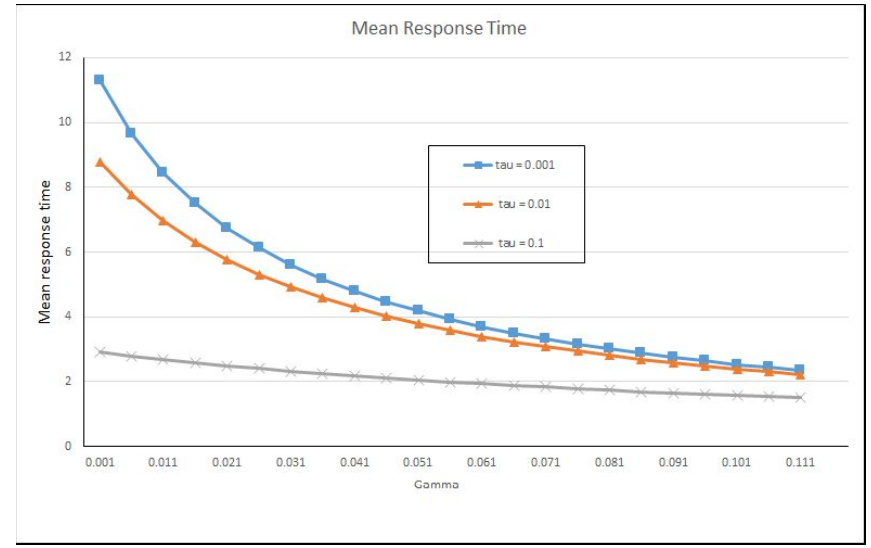

Fig. 5. Mean response time vs. failure rate

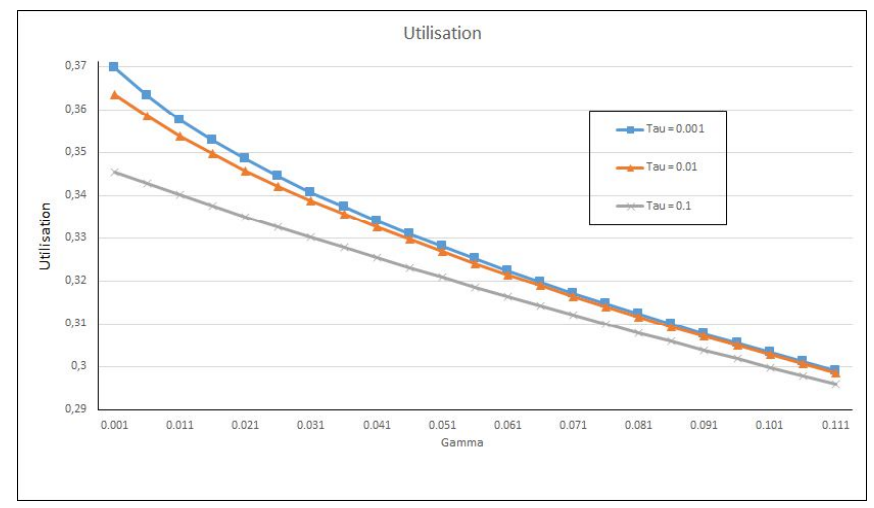

Fig. 6. Utilization vs. failure rate

been investigated. The impatient property and the catastrophic breakdowns result extra dimension in the Kolmogorov balance equations, so the recursive numerical solution can not be provided. The MOSEL-2 tool was used for solving the system equations and calculating the steady-state probabilities. Due to the finite state space, these probabilities exist, there are no stability problems. With the help of the system probabilities, reliability investigations can be performed. In this paper, the most important and interesting system measures were presented. A lot of system parameters were tried. Those parameters were chosen, where the considered performance measures display significant deviation between the scenarios.

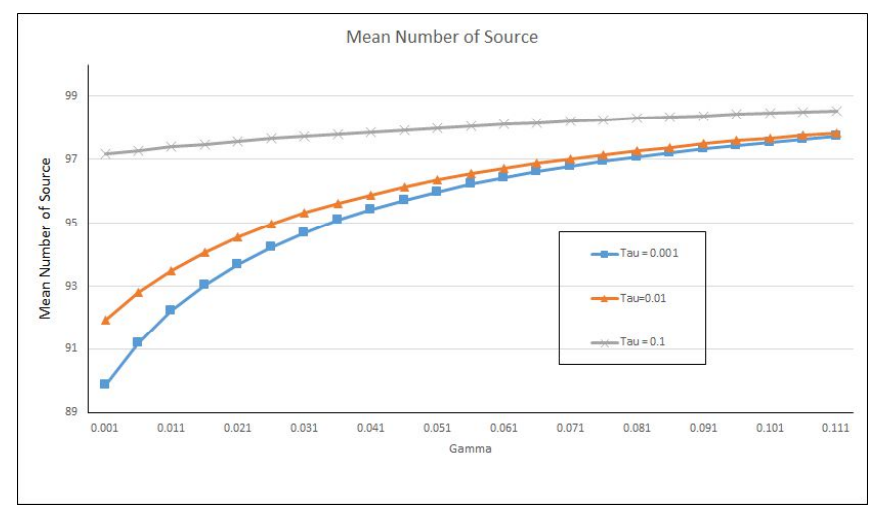

Fig. 7. Mean number of source vs. failure rate 


\section{ACKNOWLEDGMENT}

The research work was supported by the construction EFOP - 3.6.3 - VEKOP - 16-2017-00002. The project was supported by the European Union, co-financed by the European Social Fund.

The research work was supported by the Austro-Hungarian Cooperation Grant No 106öu4, 2020.

\section{REFERENCES}

[1] V. Anisimov, "Asymptotic analysis of highly reliable retrial systems with finite capacity," In: Queues, Flows, Systems, Networks. Proceedings of the International Conference Modem Mathematical Methods of Investigating the Telecommunicational Networks, pp. 7-12, 1999.

[2] J. Artalejo and A. G. Corral, Retrial Queueing Systems: A Computational Approach. Springer, 2008.

[3] J. Wang, L. Zhao, and F. Zhang, "Analysis of the finite source retrial queues with server breakdowns and repairs," Journal of Industrial and Management Optimization, vol. 7, no. 3, pp. 655-676, 2011.

[4] V. I. Dragieva, "A finite source retrial queue: number of retrials," Communications in Statistics-Theory and Methods, vol. 42, no. 5, pp. 812-829, 2013.

[5] J. Kim and B. Kim, "A survey of retrial queueing systems," Annals of Operations Research, vol. 247, no. 1, pp. 3-36, 2016.

[6] B. Almási, T. Bérczes, A. Kuki, J. Sztrik, and J. Wang, "Performance modeling of finite-source cognitive radio networks." Acta Cybern., vol. 22, no. 3, pp. 617-631, 2016.

[7] B. Almási, J. Roszik, and J. Sztrik, "Homogeneous finite-source retrial queues with server subject to breakdowns and repairs." Math. Comput. Modelling, vol. 42, no. 5-6, pp. 673-682, 2005.

[8] V. I. Dragieva, "Number of retrials in a finite source retrial queue with unreliable server." Asia-Pac. J. Oper. Res., vol. 31, no. 2, p. 23, 2014.

[9] N. Gharbi and C. Dutheillet, "An algorithmic approach for analysis of finite-source retrial systems with unreliable servers," Computers \& Mathematics with Applications, vol. 62, no. 6, pp. 2535-2546, 2011.

[10] F. Zhang and J. Wang, "Performance analysis of the retrial queues with finite number of sources and service interruptions," Journal of the Korean Statistical Society, vol. 42, no. 1, pp. 117-131, 2013.

[11] A.-A. Ali and S. Wei, "Modeling of coupled collision and congestion in finite source wireless access systems," in Wireless Communications and Networking Conference (WCNC), 2015 IEEE. IEEE, 2015, pp. 1113-1118.

[12] S. Balsamo, G.-L. Dei Rossi, and A. Marin, "Modelling retrial-uponconflict systems with product-form stochastic Petri nets," in International Conference on Analytical and Stochastic Modeling Techniques and Applications. Springer, 2013, pp. 52-66.

[13] A. Kvach and A. Nazarov, Sojourn Time Analysis of Finite Source Markov Retrial Queuing System with Collision. Cham: Springer International Publishing, 2015, ch. 8, pp. 64-72.

[14] A. Nazarov, A. Kvach, and V. Yampolsky, Asymptotic Analysis of Closed Markov Retrial Queuing System with Collision. Cham: Springer International Publishing, 2014, ch. 1, pp. 334-341.

[15] T. V. Lyubina and A. A. Nazarov, "Research of the non-markov dynamic retrial queue system with collision (in russian)," Herald of Kemerovo State University, vol. 1, no. 49, pp. 38-44, 2012.

[16] Y. Peng, Z. Liu, and J. Wu, "An M/G/1 retrial G-queue with preemptive resume priority and collisions subject to the server breakdowns and delayed repairs." J. Appl. Math. Comput., vol. 44, no. 1-2, pp. 187-213, 2014.

[17] S. Subramanian et al., "A stochastic model for automated teller machines subject to catastrophic failures and repairs," Queueing Models and Service Management, vol. 1, no. 1, pp. 75-94, 2018.

[18] B. Thilaka, B. Poorani, and S. Udayabaskaran, "Performance analysis for queueing systems with close down periods subject to catastrophe," International Journal of Pure and Applied Mathematics, vol. 119, no. 7, pp. 39-57, 2018.

[19] U. Gupta, N. Kumar, and F. Barbhuiya, "A queueing system with batch renewal input and negative arrivals," in Applied Probability and Stochastic Processes. Springer, 2020, pp. 143-157.

[20] D. Piriadarshani, S. Narasimhan, M. Maheswari, B. James et al., "A retrial queuing system operating in a random environment subject to catastrophes," European Journal of Molecular \& Clinical Medicine, vol. 7, no. 2, pp. 5029-5032, 2020.
[21] S. I. Ammar, A. Zeifman, Y. Satin, K. Kiseleva, and V. Korolev, "On limiting characteristics for a non-stationary two-processor heterogeneous system with catastrophes, server failures and repairs," Journal of Industrial \& Management Optimization, vol. 17, no. 3, p. 1057, 2021. 